and

$$
\int_{-\infty}^{\infty} V^{-1} z^{2} d x=\int_{a}^{b} V^{-1} z^{2} d x \leqq \int_{a}^{b} V^{-1} d x / 4(b-a) .
$$

Hence, the expression [ $\cdots]$ of (17) is, for $g=z$, majorized by const. $(b-a)^{-3} \int_{a}^{b} V^{-1} d x$. It is clear that each $z$ can be smoothed out so as to obtain a function $g$ possessing continuous second derivatives of the type allowed in (17) and such that $[\cdots]$ has again the same majorant. Condition (16) now yields (17) and the proof of the Theorem is complete.

\title{
REFERENCES
}

1. N. Aronszajn, On a problem of Weyl in the theory of singular Sturm-Liouville equations, Am. J. Math. 79, 597-610 (1957)

2. J. M. Cook, Convergence to the Moller wave-matrix, J. Math. and Phys. 36, 82-87 (1957)

3. K. O. Friedrichs, Über die Spektralzerlegung eines Integraloperators, Math. Ann. 115, 249-272 (1938)

4. K. O. Friedrichs, On the perturbation of continuous spectra, Communs. Appl. Math. 1, 361-401 (1948)

5. K. O. Friedrichs, Mathematical aspects of the quantum theory of fields, Interscience Publ., New York, 1953.

6. M. N. Hack, On convergence to the Moller wave operators, Nuovo Cimento 9, 731-733 (1958)

7. J. M. Jauch, Theory of the scattering operator, Helv. Phys. Acta 31, 127-158 (1958)

8. J. M. Jauch and I. I. Zinnes, The asymptotic condition for simple scattering systems, Nuovo Cimento 11, 553-567 (1959)

9. T. Kato, On finite-dimensional perturbations of self-adjoint operators, J. Math. Soc. Japan 9, 239-249 (1957)

10. T. Kato, Perturbation of continuous spectra by trace class operators, Proc. Japan Acad. 33, 260-264 (1957)

11. K. Kodaira, The eigenvalue problem for ordinary differential equations of the second order and Heisenberg's theory of S-matrices, Am. J. Math. 71, 921-945 (1949)

12. S. T. Kuroda, On the existence and the unitary property of the scattering operator, Nuovo Cimento 12, 431-454 (1959)

13. C. R. Putnam, Commutators and absolutely continuous operators, Trans. Am. Math. Soc. 87, 513-525 (1958)

14. C. R. Putnam, Commutators, perturbations, and unitary spectra, Acta Mathematica (to appear)

15. M. Rosenblum, Perturbation of the continuous spectrum and unitary equivalence, Pacific J. Math. 7, 997-1010 (1957)

16. E. C. Titchmarsh, Eigenfunction expansions associated with second-order differential equations, Oxford, 1946

17. A. Winter, Small perturbations, Am. J. Math. 67, 417-430 (1945)

18. H. Weyl, Über gewöhnliche Differentialgleichungen mit Singularitäten und die zugehörigen Entwicklungen willkürlicher Funktionen, Math. Ann. 68, 220-269 (1910)

\section{AN UPPER BOUND ON NON-NEGATIVE TRANSIENT RESPONSES*}

\author{
BY
}

\section{A. H. ZEMANIAN (New York University)}

In a recent note [1], it was shown that, if the real-valued function $w(t)$ of the real variable $t$ is zero for $t<0$ and if its Laplace transform $W(s)$ is given by

$$
W(s)=\frac{a_{n} s^{n}+a_{n-1} s^{n-1}+\cdots+a_{0}}{s^{m}+b_{m-1} s^{m-1}+\cdots+b_{0}}=\frac{N(s)}{D(s)},
$$

where $m \geq 2 n$ and the real parts of the roots of the polynomial $D(s)$ are all non-positive,

${ }^{*}$ Received July 5, 1961. 
then for $t>0$

$$
|w(t)| \leq \frac{\left|a_{n}\right| t^{m-n-1}}{(m-n-1) !}+\frac{\left|a_{n-1}\right| t^{m-n}}{(m-n) !}+\cdots+\frac{\left|a_{0}\right| t^{m-1}}{(m-1) !}
$$

If we replace the restriction that $m \geq 2 n$ by the condition that $w(t) \geq 0$, a similar inequality results and it has a very simple proof.

More specifically, if $w(t)$ is a real-valued non-negative function of the real variable $t$ and is zero for $t<0$ and if its Laplace transform is given by (1) where $m>n$ and the roots of the polynomial $D(s)$ have non-positive real parts, then for $t>0$

$$
w(t) \leq \frac{a_{n} t^{m-n-1}}{(m-n-1) !}+\frac{a_{n-1} t^{m-n}}{(m-n) !}+\cdots+\frac{a_{0} t^{m-1}}{(m-1) !} .
$$

To establish this result, let

$$
F(s)=\frac{a_{n} s^{n}+\cdots+a_{0}}{s^{m}+\cdots+b_{0}} \cdot \frac{s^{m}+\cdots+b_{0}}{s^{m}} .
$$

The corresponding inverse Laplace transform that is zero for $t<0$ may be written as follows for $t>0$.

$$
\begin{aligned}
f(t)= & \frac{a_{n} t^{m-n-1}}{(m-n-1) !}+\cdots+\frac{a_{0} t^{m-1}}{(m-1) !} \\
= & w(t)+b_{m-1} \int_{0}^{t} w(x) d x+\cdots \\
& \quad+\frac{b_{0}}{(m-1) !} \int_{0}^{t}(t-x)^{m-1} w(x) d x .
\end{aligned}
$$

Since the roots of $D(s)$ are real or appear in complex-conjugate pairs and their real parts are all non-positive, every $b_{i}$ is non-negative. Thus, all terms on the right-hand side of (4) are non-negative so that (4) implies (3).

\section{REFERENCE}

1. A. H. Zemanian, Further properties of certain classes of transfer functions: II, Quart. Appl. Math. $19,158-159$ (1961)

\section{AN APPLICATION OF THE EULER-MACLAURIN SUM FORMULA TO OPERATIONAL MATHEMATICS*}

By IRVING FRANK (Avco-Everett Research Laboratory, Everett, Mass.)

Introduction. The use of operational methods is especially well adapted to the solution of various problems in applied mathematics. Thus, in problems on heat conduction one is often enabled to find special solutions of the heat balance equation suitable for large or small values of time. It is shown in the present study that the Euler-Maclaurin sum formula may be used in some cases to generate approximate solutions to the heat balance equation which give good results for all values of time, using only a limited number of terms.

${ }^{*}$ Received July 24, 1961. 